\title{
Comparative Performance Analysis of LMS and NLMS on ECG Signal by using TMS320C6713 DSK Kit
}

\author{
Chaitanya M. Patil \\ Department of Electronics and Telecommunication \\ K.J. Somaiya College Of Engineering \\ Mumbai, Vidyavihar
}

\author{
R.A. Deshpande \\ Department of Electronics and Telecommunication \\ K.J. Somaiya College Of Engineering \\ Mumbai, Vidyavihar
}

\begin{abstract}
Adaptive filtering constitutes one of the core technologies in the field of digital signal processing and finds numerous application in the areas of science and technology viz. echo cancellation, channel equalization, adaptive noise cancellation, adaptive beam-forming, biomedical signal processing etc. Noise of ECG signal will minimize by using two algorithms that is LMS and NLMS on TMS320C6713 DSK kit. A Simulink model is designed to generate auto C code for the DSP processor. The output results are analyzed using Digital Storage Oscilloscope (DSO) in terms of filtered signal. The results have also been compared with the LMS algorithm to prove the superiority of NLMS algorithm.
\end{abstract}

\section{General Terms}

Adaptive filter, Least mean square algorithm (LMS), Normalized least mean square algorithm (NLMS).

\section{Keywords}

Adaptive filter;Least Mean Squared; Normalized Least Mean Squared; ECG signal;Digital Storage Oscilloscope(DSO);TMS320C6713 DSK kit

\section{INTRODUCTION}

The distinguishing feature of adaptive filters is that they can modify their response to improve their performance during operation without any intervention from the user [1]. The purpose of an adaptive filter is to remove noise from a signal adaptively to improve the signal to noise ratio. Figure 1 shows the diagram of a typical adaptive filter use for noise cancellation

The discrete adaptive filter process the reference signal $\mathrm{n} 1(\mathrm{n})$ to produce the output signal $\mathrm{y}(\mathrm{n})$ by a convolution with filter's weight, $w(n)$. Then, a desired signal reference signal $d(n)$ is compared with the output $y(n)$ to obtain an estimation error $\mathrm{e}(\mathrm{n})$.The objective here is to minimize the error signal e(n) which is equivalent to minimize the error signal $\mathrm{e}(\mathrm{n})$ which is equivalent to minimize $d(n)-y(n)$. This error signal is used to incrementally adjust the filter's weights for next time instant. The algorithms for weight update are: wiener filter, leastmean square (LMS) algorithm, Normalized-least-mean square(NLMS) algorithm, recursive-least-square(RLS) algorithm, and kalman filter. Rest of the paper is organized as follows

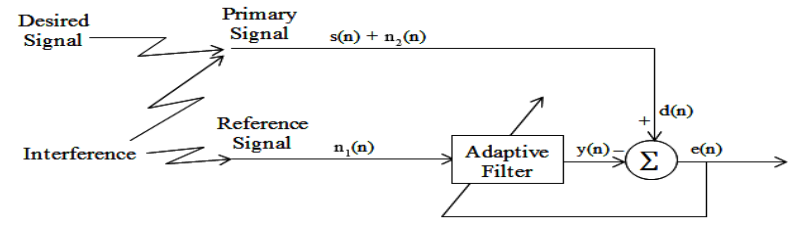

Fig1: Adaptive filter for noise cancellation [1]

\section{RELATED WORK}

Schutz [2, 7] discussed the model based design of acoustic noise cancellation using Simulink on TI C6416 platform. Jan vanus [3] describes a proposition of the method for optimal adjustment parameters of the adaptive filter with LMS algorithm. [4] Results reveal that the LMS and NLMS are better than the other adaptive filters. However, the obtained SNR of reconstructed signal of LMS filter is lower than that of the NLMS filter. So, the NLMS adaptive filter is more appreciable for removing the power line interference from the ECG signal.

\section{ADAPTIVE FILTER ALGORITHMS}

\subsection{Least mean Square Algorithm}

First, The filter tap weights of the adaptive filter are updated according to the following formula: $\mathrm{w}(\mathrm{n}+1)=\mathrm{w}(\mathrm{n})+2 \mu \mathrm{e}(\mathrm{n}) \times(\mathrm{n})$;

Wherex(n) is the input vector of time delayed input values, and is given by

$$
\begin{aligned}
& x(n)=[x(n) x(n-1) x(n-2) \ldots x(n-N+1)] T \\
& \& w(n)=[w 0(n) w 1(n) w 2(n) \ldots . w N-1(n)] T
\end{aligned}
$$

Represents the coefficients of the adaptive FIR filter tap weight vector at time $n$ and $\mu$ is known as the step size parameter and is a small positive constant.

\subsection{Derivation of Least mean Square Algorithm}

Based on wiener solution. Also Depends on Steepest Descent Algorithm

$\mathrm{w}(\mathrm{n}+1)=\mathrm{w}(\mathrm{n})-(\mu / 2) \nabla \xi(\mathrm{n}) ;$

where $\xi(n)=E\left[e(n)^{\wedge} 2\right]$ is a cost function.

The gradient of the cost function, $\xi(n)$ can alternatively be expressed in the following form:

$\nabla \xi(\mathrm{n})=\nabla\left(\mathrm{e}(\mathrm{n})^{\wedge} 2\right)$

$$
=\partial\left(\mathrm{e}(\mathrm{n})^{\wedge} 2\right) / \partial \mathrm{w}
$$

By solving it by wiener solution, we get;

$\nabla \xi(\mathrm{n})=-2 \mathrm{e}(\mathrm{n}) \mathrm{x}(\mathrm{n})$

Substituting this into the steepest descent algorithm, we arrive at the recursion for the LMS adaptive algorithm. $\mathrm{w}(\mathrm{n}+1)=\mathrm{w}(\mathrm{n})+\mu \mathrm{e}(\mathrm{n}) \mathrm{x}(\mathrm{n})$.

\subsection{Implementation of Least mean Square Algorithm}

For the Implementation of each iteration of the LMS algorithm requires three distinct steps in the following order: 
1. The output of the FIR filter,

$\mathrm{y}(\mathrm{n})=\mathrm{wT}(\mathrm{n}) \mathrm{x}(\mathrm{n})$

2. The value of the error estimation is calculated using, $\mathrm{e}(\mathrm{n})=\mathrm{d}(\mathrm{n})-\mathrm{y}(\mathrm{n})$

3. The tap weights of the FIR vector are updated in preparation for the next iteration,

$\mathrm{w}(\mathrm{n}+1)=\mathrm{w}(\mathrm{n})+2 \mu \mathrm{e}(\mathrm{n}) \mathrm{x}(\mathrm{n})$

For each iteration, the LMS algorithm requires $2 \mathrm{~N}$ additions and $2 \mathrm{~N}+1$ multiplication.

\subsection{Normalized Least mean Square Algorithm}

When the convergence factor $\mu$ is large, the algorithm experiences a gradient noise amplification problem. In order to solve this difficulty we can use the NLMS algorithm. The correction applied to the weight vector $w(n)$ at iteration $n+1$ is "normalized" with respect to the squared Euclidian norm of the input vector $\mathrm{x}(\mathrm{n})$ at iteration $\mathrm{n}$. Convergence factor $\mu$ -

$\mu(\mathrm{n})=\alpha /\left(\mathrm{c}+\|\mathrm{x}(\mathrm{n})\|^{\wedge} 2\right) ;$

where $\mathrm{c}$ is constant less than 1 , and $\alpha$ is NLMS adaption constant and should satisfy $0<\alpha<2$. It obeys principle of minimum disturbance

\subsection{Derivation of Normalized Least mean square algorithm}

To derive the NLMS algorithm we consider the standard LMS recursion in which we select a variable step size parameter, $\mu(n)$. This parameter is selected so that the error value, $e+(n)$, will be minimized using the updated filter tap weights, $\mathrm{w}(\mathrm{n}+1)$, and the current input vector, $\mathrm{x}(\mathrm{n})$

The filter weight updated by;

$$
\mathrm{w}(\mathrm{n}+1)=\mathrm{w}(\mathrm{n})+\left\{\alpha /\left(\mathrm{c}+\|\mathrm{x}(\mathrm{n})\|^{\wedge} 2\right)\right\} \mathrm{e}(\mathrm{n}) \mathrm{x}(\mathrm{n})
$$

\subsection{Implementation of Normalized Least mean square algorithm}

It is essentially an improvement over LMS algorithm with the added calculation of step size parameter for each iteration.

1. The output of the adaptive filter is calculated as:

$\mathrm{y}(\mathrm{n})=\mathrm{w} T(\mathrm{n}) \mathrm{x}(\mathrm{n})$

2. The error signal is calculated as the difference between the desired output and the filter output given by:

$\mathrm{e}(\mathrm{n})=\mathrm{d}(\mathrm{n})-\mathrm{y}(\mathrm{n})$

3. The step size and filter tap weight vectors are updated using the following equations in preparation for the next iteration:

$$
\text { For } \mathrm{i}=0,1,2, \ldots \ldots . \mathrm{N}-1 \text {; }
$$

$\mu \mathrm{i}(\mathrm{n})=\alpha /\left(\mathrm{c}+\|\mathrm{xi}(\mathrm{n})\|^{\wedge} 2\right)$

With $\alpha=0.02$ and $c=0.001$, each iteration of the NLMS algorithm requires $3 \mathrm{~N}+1$ multiplication operations.

\section{EXPERIMENTAL SETUP}

In this we required the recorded ECG signal, TMS320C6713 DSK kit and digital storage oscilloscope as output to display the output waveform. Additional noise reference input is also applied at line-in port. The input signals are processed by DSK that is controlled by simulink model running in the computer system. The Adaptive Noise Cancellation (ANC) Simulink model is designed using LMS and NLMS algorithms for generating $\mathrm{C}$ code and to download this code on DSP target processor. The ANC model is designed with help of inbuilt library of Simulink and the blocks are reconfigured as per the requirements of TMS320C6713 DSP processor.

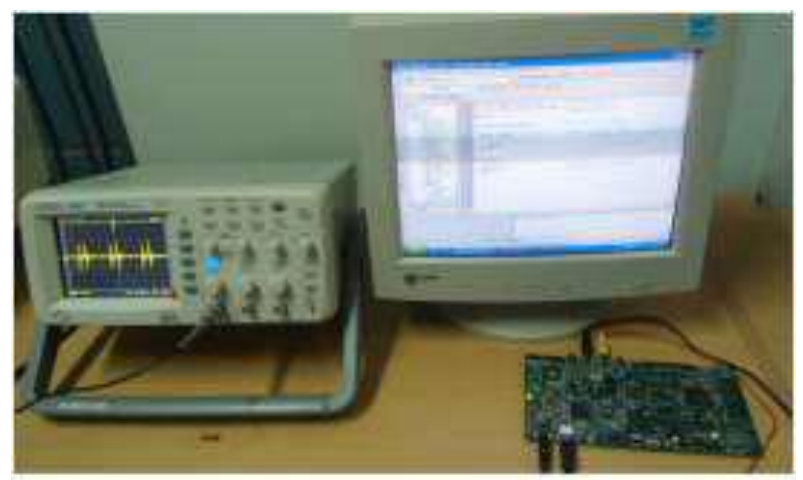

Fig2: Experimental Setup



Fig3: Simulink model for LMS filter algorithm

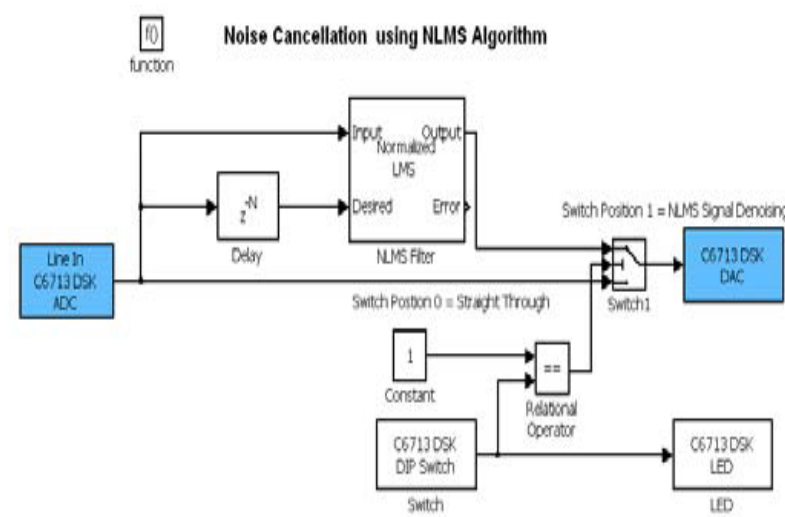

Fig4: Simulink model for NLMS filter algorithm

The figure 2 shows experimental setup for the project. Then in figure 3 and figure 4 Simulink model is shown.

\section{RESULTS}

The real-time implementation is also done to remove the noise of an ECG signal which is corrupted by various types of interferences \& distortions. Figure 5 shows de-noise ECG signal by LMS filter algorithm. 


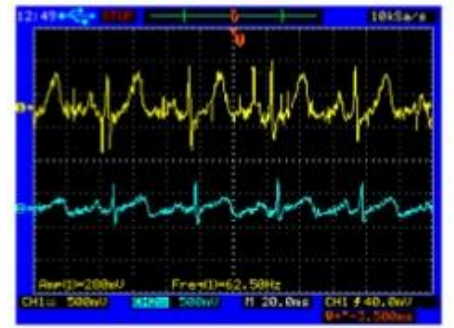

Fig5: De-noise ECG signal by NLMS filter

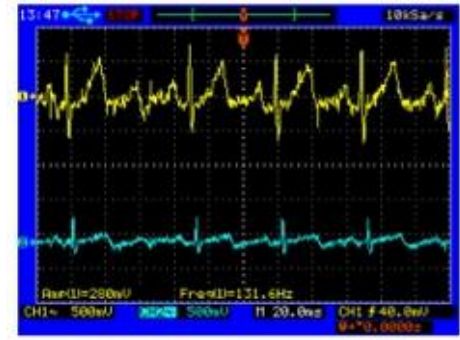

Fig6: De-noise ECG signal by LMS filter

Table 1 . SNR Improvement in LMS and NLMS in dB

\begin{tabular}{|l|l|l|l|}
\hline Sr. no. & $\begin{array}{l}\text { Noise } \\
\text { variance }\end{array}$ & $\begin{array}{l}\text { SNR } \\
\text { improvement } \\
\text { (LMS) }\end{array}$ & $\begin{array}{l}\text { SNR } \\
\text { improvement } \\
\text { (NLMS) }\end{array}$ \\
\hline 1 & 0.03 & 6.2 & 7.4 \\
\hline 2 & 0.75 & 5 & 6.11 \\
\hline
\end{tabular}

\section{CONCLUSION}

The implementation of LMS \&NLMS on DSK TMS320C6713 hardware for an ECG signal has been done successfully and the results are compared in the terms of SNR Improvement. During the simulation for NLMS algorithm, we got the SNR Improvement of 6.11dB. During the simulation for LMS algorithm, we got the SNR improvement of $5 \mathrm{~dB}$. By using NLMS algorithm which highly preserves the peak of QRS complex of ECG signal compared to LMS algorithm. Hence we conclude that NLMS algorithm is better than that of LMS algorithm.

\section{ACKNOWLEDGEMENT}

I would like to thank the all authors who earlier work on adaptive filter whose guidance significantly contributed to the work made in this paper.

\section{REFERENCES}

[1] Googleimage"https://www.google.co.in/search?q=adapti ve + filter".

[2] Schutz K. "Code verification using RTDX".Available at MathWorksMatlab Central File Exchange.

[3] Jan vanus,Application of optimal setting of the LMS adaptive filter for speech signal processing" Proc.InternationalMulticonference on Computer Science and Information Technology pp.767-774.

[4] Md. Moniruzzaman,"least-mean-square algorithm based adaptive filters for removing power line interference from ECG signal" Proc.IEEE international conference on informatics,electronics\& vision.

[5] Bernard Widrow, John R. Glover, John M. Mccool, John Kaunitz, Charles S. Williams, Robert H. Hean, James R. Zeidler, Eugene Dong,Jr. and Robert C. Goodlin, “Adaptive Noise Cancelling: Principles and Applications ",Proceedings of the IEEE, 1975, Vol.63 , No. 12 , Page(s): $1692-1716$.

[6] Abutaleb, A.S, "An adaptive filter for noise cancelling", IEEE Transactions on, Circuits and Systems, 1988, Vol.35, No.10, Page(s):1201 - 1209.

[7] SchutzK.,"Using the Target/Host RTDX blocks to implement an acoustic noise canceller". Available at MathWorkMatlab Central File Exchange

[8] Texas Instruments Tutorial,"TMS320C6713 Hardware Designers Resource Guide”, (July 2004), SPRAA33.

[9] Raj Kumar Thenua, AasthaKatara, D.C. Dhubkaria,"Simulation of Adaptive Noise Canceller for an ECG signal Analysis", in an International Conference on Advances in Computer Science (ACS) organized by Engineers Networks at Noida, India during 20-21 Dec2011 